\title{
AVALIAÇÃO DA SIMETRIA E DESCARGA DE PESO ENTRE OS MEMBROS INFERIORES DE ATLETAS DE FUTEBOL DA CATEGORIA DE BASE
}

\author{
EVALUATION OF SYMMETRY AND WEIGHT-BEARING IN LOWER LIMBS OF YOUTH SOCCER PLAYERS \\ EVALUACIÓN DE LA SIMETRÍA Y DE LA DESCARGA DE PESO EN LOS MIEMBROS INFERIORES DE \\ ATLETAS DE FÚTBOL DE LA CATEGORÍA DE BASE
}

Artigo Original

Daniel Lucas Spagnuolo
(Fisioterapeuta) $^{1}$
Felipe Alves Machado
(Fisioterapeuta) $^{2}$
Maria Stella Peccin (Fisioterapeuta) $^{3}$

1. Universidade Federal de São

Paulo - Santos, SP, Brasil.

2. Centro Universitário Metodista IPA - Porto Alegre, RS, Brasil.

3. Faculdade de Ciências da Saúde do Instituto de Porto Alegre -

Porto Alegre, RS, Brasil.

\section{Correspondência:}

Avenida Professor Francisco Morato,

2.203, 23/A3, Caxingui - 05513-300

São Paulo, SP, Brasil.

dls874@hotmail.com

\section{RESUMO}

Introdução: A avaliação instrumentada dos saltos verticais informa sobre as capacidades funcionais e variáveis neuromusculares, auxiliando no atendimento dos atletas. Essencial dar atenção maior para as categorias de base, pois contém fases importantes do desenvolvimento dos atletas que serão o futuro do esporte. Objetivo: Avaliar as características funcionais e neuromusculares de atletas de futebol da categoria de base, no desempenho em saltos verticais. Metodologia: Foram avaliados atletas de futebol com idade entre 15 e 17 anos. Foram realizados em duas plataformas de força os seguintes saltos verticais: salto em agachamento, salto em contramovimento, salto pliométrico de $40 \mathrm{~cm}$ de altura. Para análise das variáveis das forças concêntrica, excêntrica, impacto e altura dos saltos utilizou-se a prova estatística de Shapiro-Wilk, descrição das variáveis em média e desvio padrão, considerando quando $p \leq 0,05$. Resultados: Dos 30 atletas avaliados, obtivemos: três goleiros (10\%), cinco zagueiros (16,7\%), um lateral (3,3\%), três volantes (10\%), 12 meio-campistas (40\%) e seis atacantes (20\%). A categoria sub-17 apresentou maiores resultados comparada a categoria sub-15. Maior altura de salto do contramovimento em relação ao salto em agachamento $(p<0,05)$ e ao pliométrico $(p>0,05)$. Sem diferença na força concêntrica entre as categorias e os tipos de saltos. Maior força de impacto no contramovimento. Maior força em membro inferior direito em todos os saltos e variáveis de força. Conclusões: Encontramos assimetria entre os membros inferiores, podendo correlacionar com um déficit de potência muscular, má desaceleração do movimento e erros biomecânicos. Fatores predisponentes a lesões musculoesqueléticas, os quais podem ser prevenidos. A avaliação instrumentada da força através desses testes pode subsidiar os profissionais do clube como uma ferramenta de trabalho, capaz de aprimorar o treinamento, preparação e reabilitação. Entretanto, são necessárias mais pesquisas, de forma que momentos distintos da idade e da temporada do atleta sejam avaliados.

Palavras-chave: esportes, prevenção e controle, lesões.

\section{ABSTRACT}

Introduction: The instrumented jump tests evaluation inform us about the functional capabilities and neuromuscular variables assisting in the care of athletes. It is essential to give a greater attention to the basic categories because it contains important stages of development of the athletes who are the future of the sport. Objective: To evaluate the functional and neuromuscular youth soccer athletes' category based on performance in jump tests. Methods: Soccer players aged between 15 and 17 years were evaluated. The following jump tests were performed on two force platforms: squat Jump; countermovement test; drop jump of $40 \mathrm{~cm}$. To analyze the variables concentric and eccentric forces, impact and height of jumps, the Shapiro-Wilk test, mean and standard deviation of variables with $p \leq 0.05$ were used. Results: Of the 30 athletes evaluated, we found: three goalkeepers (10\%), five quarterbacks (16.7\%), one winger (3.3\%), three defensive midfielders (10\%), 12 midfielders (40\%) and six strikers (20\%). The under 17 category had higher results compared to the under 15;it was achieved maximum height of the countermovement jump over the squat jump ( $p$ $<0.05$ ) and drop jump ( $p>0.05)$; no difference was found in concentric force between the categories and types of jumps; greater impact force was obtained on countermovement jump and an increased strength in the right lower limb was found in all jump tests and variables. Conclusions: We found an asymmetry between the lower limbs, which may correlate with a muscle power deficit, poor eccentric movement and biomechanical errors. These predisposing factors for musculoskeletal injuries can be prevented. The instrumented evaluation of muscle force through these tests can assist club professionals as a working tool, able to enhance training programs, preparation and rehabilitation. However, more research is needed in order to evaluate different moments of the athlete's age and season.

Keywords: sports, prevention \& control, injuries.

\section{RESUMEN}

Introducción: La evaluación por instrumentos de los saltos verticales suministra informaciones sobre las capacidades funcionales y las variables neuromusculares, ayudando para los cuidados de los atletas. Es esencial dar más atención a las categorías de base, pues contienen fases importantes del desarrollo de los atletas, quienes serán el futuro 
del deporte. Objetivo: Evaluar las características funcionales y neuromusculares de atletas de fútbol de la categoría de base, en el desempeño cuanto a saltos verticales. Metodología: Se evaluaron atletas de fútbol de edades entre 15 y 17 años. Se realizaron, en dos plataformas de fuerza, los siguientes saltos verticales: salto en agachamento, salto en contramovimiento, salto pliométrico de $40 \mathrm{~cm}$ de altura. Para el análisis de las variables referentes a las fuerzas concéntrica, excéntrica, impacto y altura de los saltos, se utilizó la prueba estadística de Shapiro-Wilk, descripción de las variables en promedio y desvío estándar, considerando cuando $p \leq 0,05$. Resultados: De los 30 atletas evaluados, identificamos: tres arqueros (10\%), cinco zagueros (16,7\%), un lateral (3,3\%), tres volantes (10\%), 12 mediocampistas (40\%) y seis delanteros (20\%). La categoría sub-17 presentó resultados mejores en comparación con la categoría sub-15; más altura de salto del contramovimiento en relación con el salto en agachamento $(p<0,05)$ y al pliométrico ( $p>0,05)$; sin diferencia en la fuerza concéntrica entre las categorías y los tipos de saltos. Más fuerza de impacto en el contramovimiento; más fuerza en el miembro inferior derecho en todos los saltos y variables de fuerza. Conclusiones: Encontramos asimetría entre los miembros inferiores, pudiéndose correlacionar con un déficit de potencia muscular, mala desaceleración del movimiento y errores biomecánicos. Se hallaron factores que predisponen a lesiones musculoesqueléticas, los cuales pueden ser prevenidos. La evaluación, por instrumentos, de la fuerza, mediante estas pruebas, puede ayudar a los profesionales del club como una herramienta de trabajo que puede perfeccionar el entrenamiento, la preparación y la rehabilitación. No obstante, se necesitan más investigaciones, de modo que sean evaluados momentos diferentes de la edad y la temporada del respectivo atleta.

Palabras clave: deportes, prevención y control, lesiones.

Artigo recebido em 17/03/2013, aprovado em 26/09/2013.

\section{INTRODUÇÃO}

O esporte mais popular do mundo, futebol, conta com 203 países associados à FIFA (Federation International Football Association). Contendo por volta de 200 milhões de praticantes ${ }^{1-3}$. Devido uma mudança de foco no futebol, passando do futebol-arte, conhecido da época de Pelé e Maradona, para futebol de marcação, surgiram jogadas mais agressivas, o que pode relacionar-se com o aumento de casos de lesões, principalmente muscular .

Lesão, que por definição é caracterizada quando ocorre um afastamento do treinamento ou jogo, necessidade de atendimento médico ou fisioterapêutico, diagnóstico de dano tecidual além de qualquer subdivisão desses critérios. Sendo mais recorrentes em atletas homens adultos do que em mulheres e adolescentes ${ }^{3}$.

Estudos mostram que a posição do atleta (goleiro, meio-campista, atacante etc) não interfere na incidência de lesões, mas nos jogos há maior propensão à ocorrência de lesões em relação aos treinos. Sendo que essas lesões acometem principalmente as pernas (joelhos) e é de caráter traumático por contato físico ${ }^{4-6}$. Entretanto, Hägglund et al., em 2006, não relataram diferenças significativas entre lesões em treinos e jogos entre duas temporadas em um estudo prospectivo ${ }^{7}$.

Hoje em dia a prevenção tem sido aplicada no futebol, mostrando a importância desse tipo de trabalho principalmente para essas regiões, bem como as lesões musculares e traumatismos. A simples aplicação de um questionário pode identificar esses principais focos de lesão ${ }^{2,8}$. A prevenção, em mais de 20 anos, nunca foi o foco principal dos estudos com futebol. Entretanto, Ekstrand et al., em 1983, mostraram redução significante de entorses de tornozelo e joelho, aplicando um programa de prevenção $0^{5,8,9}$.

Além das disfunções articulares, como desequilíbrio da contração muscular, fadiga e alterações inflamatórias que influem na coordenação muscular, provocando dor e consequentemente incapacidade funcional ${ }^{10}$, idade, sexo, nível de treinamento e, principalmente, lesões precedentes podem estar ligados à ocorrência das lesões esportivas, necessitando de treinamento e atenção especial a esses atributos nos atletas. Contando também com um programa de atendimento de uma equipe interdisciplinar ${ }^{1,7}$.

Trabalhos de aquecimento e um resfriamento pós-atividade, assim como exercícios de melhora da coordenação, tempo de reação e resistência mostraram ser efetivos para a prevenção de lesões, diminuindo a incidência em relação a um grupo controle, no qual não foram feitos esses trabalhos preventivos. Estudos confirmam a importância da atuação dos profissionais da saúde antes que aconteça a lesão, diminuindo a ida de atletas ao setor de reabilitação e departamento médico, tempo de afastamento e melhora no desempenho do atleta2,8.

As distensões musculares são mais presentes nos músculos isquiotibiais do que nos quadríceps, por esses últimos terem grande força muscular e pelo seu tamanho. As distensões podem resultar de desequilíbrio muscular, flexibilidade precária, estiramento excessivo ou discrepâncias nos tamanhos das pernas ${ }^{11}$.

Existem diversos modos de mensurar a função muscular, desde aparelhos instrumentais a testes de campos. A implementação de um teste de avaliação tem maior confiabilidade quando simula o movimento específico da prática esportiva, tendo maior aplicabilidade, nesse caso, testes de campo em relação aos laboratoriais ${ }^{12}$.

Para determinar as habilidades físicas dos atletas, técnicos, preparadores físicos e fisioterapeutas usam os saltos verticais, servindo para melhorar treinamentos e obter parâmetro para retorno após tratamento $^{13}$. Muitas características contribuem para o desempenho nos saltos verticais, tais como produção de força muscular, técnica no salto e dados antropométricos ${ }^{14}$.

Os testes funcionais objetivos da extremidade inferior ajudam a determinar as capacidades funcionais da articulação durante atividades esportivas. Usados para determinar as limitações funcionais, que não podem ser determinadas apenas com testes musculares ${ }^{15}$. Com a evolução da tecnologia, foram criados equipamentos capazes de traduzir resultados, quantificar dados antes só analisados visualmente e, assim, mensurar mais precisamente o que se deseja. Dentre esses, a plataforma de força vem com esse intuito de incrementar as avaliações na área da saúde ${ }^{16}$.

As plataformas de força de forma retangular (com quatro sensores) são as mais comercializadas, e verificam a força de reação do solo em seus três componentes: mediolateral, anteroposterior e vertical. A força de reação do solo (FRS), decorrente do peso corporal e das magnitudes e direções impostas pelo centro de massa do corpo, é uma das mais comuns forças externas investigadas na locomoção ${ }^{17,18}$.

Com isso, achamos necessário um melhor acompanhamento e auxílio dos profissionais da área da saúde, dando uma atenção peculiar às categorias de base, as quais envolvem fases importantes do desenvolvimento do atleta. Sendo de extrema importância uma avaliação 
completa, de forma instrumentada, para melhor subsidiar a equipe de trabalho local com ferramentas para prevenir lesões, identificar riscos de recorrências, adaptar e atualizar treinamentos, e também se crie um protocolo de atendimento mais eficaz. Assim, determinamos como objetivo do trabalho a avaliação das características funcionais e neuromusculares de atletas de futebol de categoria de base, através de saltos realizados em plataformas de força.

\section{MÉTODOS}

\section{Indivíduos}

Neste trabalho avaliamos atletas das categorias de base de futebol de um clube da primeira divisão do futebol profissional do estado de São Paulo, por meio da plataforma de força, com idade entre 15 e 17 anos.

Foram selecionados atletas do gênero masculino pertencentes às categorias sub-15 e sub-17, das diversas posições de jogo (goleiro, zagueiro, lateral, volante, meio-campista e atacante). Tanto a seleção como a avaliação dos atletas foi feita conforme o responsável de cada categoria escolhia os disponíveis. As avaliações foram feitas no início da semana e antes do treinamento para que evitassem interferências do desgaste físico imposto pelo treinamento diário.

Avaliados no centro de treinamento das categorias de base do clube, no setor de fisioterapia, todos os participantes realizaram cinco minutos de bicicleta ergométrica para aquecimento prévio e preparação para os saltos. Em seguida, permaneceram três minutos em repouso para coleta de dados como nome, peso e altura, e logo após a sequência de saltos. A disponibilidade de mais indivíduos ficou impossibilitada por fatores externos, como agenda de jogos e treino do clube, diminuindo a quantidade de atletas que poderiam ser avaliados.

Foram excluídos todos os atletas que estavam em atendimento pelo departamento médico do clube, período menor que três meses de retorno à atividade da prática esportiva, falta de material adequado para realização dos saltos (tênis). Todos foram devidamente esclarecidos sobre as avaliações e, por serem menores de idade, levaram o termo de consentimento para os pais ou responsáveis assinarem. Os atletas também assinaram o termo de consentimento. O projeto de pesquisa foi aceito pelo Comitê de Ética em Pesquisa local através do número de protocolo CEP: 0680/09

\section{Plataforma de força}

Para a avaliação funcional foi utilizada a plataforma de força da marca Globus Ergo System (Treviso, Itália) que fornece dados objetivos a respeito da descarga de peso dos membros inferiores, força de reação do solo, simetria ou assimetria durante a descarga de peso dos membros inferiores, força de contração concêntrica, excêntrica e transição da fase de contração concêntrica para a excêntrica (coupling time). Foi utilizada para mensurar a qualidade de execução dos três tipos de saltos: salto em contramovimento, salto em agachamento e salto pliométrico, descritos em sessões a seguir.

As plataformas ficaram em solo plano e rígido para evitar movimentos de vibração que poderiam interferir na avaliação. Para isso, as plataformas foram acopladas a um suporte de madeira pré-moldado para encaixe das plataformas de forma que ficaram imóveis, evitando risco de entorses, alterações na coleta dos dados e análises.

Esse suporte de madeira contém as seguintes medidas: $84,5 \mathrm{~cm}$ de comprimento, $72 \mathrm{~cm}$ de largura, $11 \mathrm{~cm}$ de altura e com dois orifícios nos quais se encaixavam as plataformas de força. Foi utilizado também um banco com degrau adicional para subir no piso superior, somando uma altura total de $40 \mathrm{~cm}$, com o qual se realizou o salto pliométrico.

Cada plataforma possui dimensão de 24 x $40 \mathrm{~cm}$; conversor analógico-digital de 14 bits, frequência de amostragem de $1.000 \mathrm{c} /$ seg. Analisou-se a força em Newtons (N) de reação do solo em cada membro inferior e em cada momento do salto para força concêntrica, excêntrica de estabilização e a força de impacto após aterrissagem, além da altura desempenhada em cada tipo de salto.

\section{Salto em agachamento (squat jump)}

Neste teste o indivíduo realizou um salto vertical partindo da posição de meio-sentado (joelhos flexionados a 90) com o tronco erguido, as mãos na cintura e um pé em cada plataforma de força. Antes do início de qualquer salto, era solicitado que o atleta ficasse um tempo em cima das plataformas para cadastramento e descarga do peso em ambas as plataformas. O indivíduo efetuou o teste sem realizar contramovimentos, partindo após comando verbal do instrutor do teste. Foi solicitada a aterrissagem do salto de forma a desacelerar o movimento, realizando a tríplice flexão dos membros inferiores para que não ocorressem muita descarga e aumento da força de impactação nas articulações, principalmente do joelho. O salto é firme e realizado sem a ajuda dos braços, constitui um teste sensível de fácil aprendizado e boa padronização.

O salto em agachamento permite, mediante a altura alcançada pelo indivíduo no teste, avaliar a força explosiva dos membros inferiores. O valor da altura está diretamente relacionado com a velocidade vertical do indivíduo no momento em que a velocidade é fruto da aceleração que os membros inferiores imprimem ao centro de gravidade. Devemos lembrar que o deslocamento angular das articulações dos membros inferiores é de $90^{\circ}$ (o ângulo do joelho é igual a $180^{\circ}$ no momento mais alto do salto), valor padrão em todos os indivíduos que efetuam um salto em agachamento.

\section{Salto em contramovimento (countermovement)}

O salto de contramovimento é um teste em que a ação de saltar para cima se realiza graças ao ciclo alongamento-encurtamento. Sendo que o contramovimento se faz para baixo com uma aceleração muito modesta e os extensores de joelho se ativam apenas no momento da inversão do movimento, pode-se afirmar que o estiramento dos elementos elásticos e a sucessiva reutilização de energia elástica se fazem presentes, e que o aumento de rendimento comparado ao salto em agachamento é devido em qualquer caso ao aproveitamento do reflexo miotático (fator do tipo coordenativo).

Neste teste o indivíduo encontrou-se na posição ortostática com as mãos na cintura e realizou-se um salto vertical após o contramovimento feito para baixo (devem-se flexionar os joelhos até 90). Durante a ação de flexão o tronco permaneceu o mais erguido possível para evitar qualquer influência no rendimento dos membros inferiores. De mesma forma do salto em agachamento, o atleta só partiu para o salto após comando verbal do instrutor do teste. Foi solicitada a aterrissagem do salto de forma a desacelerar o movimento, realizando a tríplice flexão dos membros inferiores para que não ocorressem muita descarga e aumento da força de impactação nas articulações, principalmente do joelho.

\section{Salto pliométrico (drop jump)}

Neste teste o indivíduo realizou uma ação de salto vertical depois de uma queda de um banco (plinto) de $40 \mathrm{~cm}$ da altura. O indivíduo manteve as mãos na cintura e o tronco vertical para não influenciar no rendimento dos membros inferiores, e de mesma forma dos saltos anteriores, foi solicitada a aterrissagem de forma segura.

O salto pliométrico é um teste que avalia a força reativa dos membros inferiores. Neste caso a elevação do indivíduo é produzida pelo componente contrátil, pela reutilização da energia elástica acumulada durante a fase de desaceleração e pelo plus de força obtido graças à incorporação posterior de unidades motoras por via reflexa. A velocidade 
que o indivíduo possui no momento em que ele toca o chão depois da queda de $40 \mathrm{~cm}$ é de aproximadamente $3 \mathrm{~m} / \mathrm{seg}$. O indivíduo, portanto, deve anular a energia cinética depois do primeiro contato através de uma fase de contração excêntrica e, posteriormente, inverter o movimento e realizar o salto (fase de contração concêntrica).

A adição de uma altura de queda de $40 \mathrm{~cm}$ tem como finalidade poder efetuar um controle transversal entre os indivíduos que praticam futebol. A comparação dos valores de salto obtidos através do salto pliométrico e do salto em agachamento pode nos dar uma primeira visão sobre a capacidade de cada indivíduo de acumular e, posteriormente, utilizar energia elástica e também sobre a capacidade de incorporar o máximo número de unidades motoras por via reflexa.

\section{Análise estatística}

Para verificar se as variáveis mensuradas e calculadas tinham distribuição normal, empregou-se a prova estatística de Shapiro-Wilk, bem como se fez a inspeção dos gráficos de qq-plot. Uma vez que as variáveis tinham distribuição aproximada pela distribuição normal, empregaram-se métodos paramétricos para descrição e inferência estatística.

A descrição das variáveis foi realizada calculando-se a média e o desvio padrão como indicadores das medidas de tendência central e de dispersão, respectivamente. Para verificar a significância da diferença entre as categorias etárias, membro e tipo de salto para as variáveis de interesse, empregou-se a análise de variância mista em desenho misto $(2 \times 2 \times 3)$. A significância estatística foi aceita em $p \leq 0,05$.

\section{RESULTADOS}

As características antropométricas das categorias avaliadas estão apresentadas nas tabelas 1 e 2, exibidas a seguir.

Foi avaliado um total de 30 atletas com idade entre 15 e 17 anos. Todos os atletas realizaram os saltos citados acima, exceto um atleta da categoria sub-15 que não realizou o salto pliométrico (drop jump) por sentir desconforto no tornozelo direito. Com relação à posição de jogo em campo da categoria sub-15, dois são goleiros, dois zagueiros, um lateral, dois volantes, quatro meio-campistas, quatro atacantes. Na categoria sub-17 tivemos um goleiro, três zagueiros, um volante, oito meio-campistas e dois atacantes. Somando um total de três goleiros (10\%), cinco zagueiros $(16,7 \%)$, um lateral $(3,3 \%)$, três volantes $(10 \%)$, 12 meio-campistas (40\%), seis atacantes (20\%).

$\mathrm{Na}$ tabela 3 estão apresentadas as alturas dos três tipos de saltos, mostrando separadamente para cada categoria. De acordo com os resultados obtidos, independente da categoria etária, verificou-se diferença significativa $(p<0,05)$ entre os resultados obtidos para os saltos contramovimento e salto pliométrico, quando comparados ao salto em agachamento. E apesar do salto contramovimento apresentar valores brutos maiores que o salto pliométrico, esses não resultaram em uma diferença estatisticamente significativa.

Tabela 1. Características antropométricas da população estudada na categoria sub-15 ( $n=15)$.

\begin{tabular}{c|c}
\hline Variável & Média \pm desvio padrão \\
\hline Idade $(\operatorname{anos})$ & $15 \pm 0$ \\
\hline Estatura $(\mathrm{cm})$ & $176 \pm 5$ \\
\hline Peso $(\mathrm{kg})$ & $67 \pm 6$ \\
\hline
\end{tabular}

Tabela 2. Características antropométricas da população estudada na categoria sub-17 $(n=15)$

\begin{tabular}{c|c}
\hline Variável & Média \pm desvio padrão \\
\hline Idade $($ anos) & $16 \pm 0,5$ \\
\hline Estatura $(\mathrm{cm})$ & $178 \pm 6$ \\
\hline Peso $(\mathrm{kg})$ & $71 \pm 8$ \\
\hline
\end{tabular}

O perfil de força nos saltos está apresentado na tabela 4, a seguir. Detalhando em força concêntrica (Conc.), excêntrica (Exc.), impacto inicial e impacto final.

Com o objetivo de verificar se o acúmulo de energia elástica durante o salto influencia na altura do salto quando comparamos os saltos contramovimento e em agachamento, calculou-se a razão salto contramovimento por salto em agachamento, os resultados foram descritos na tabela 5. Os resultados da correlação entre as razões são apresentados na tabela 6 .

$\mathrm{Na}$ tabela 7 foram apresentados os déficits de força contralateral. Não foi possível indicar que estes eram significativamente diferentes de zero, resultado já esperado pelo observado na tabela 4.

Tabela 3. Altura de salto para os três saltos.

\begin{tabular}{c|c|c}
\hline Variável & \multicolumn{2}{|c}{ Média \pm desvio padrão } \\
\hline & SUB-15 & SUB-17 \\
\hline Salto em agachamento $(\mathrm{cm})$ & $31,33 \pm 3,18$ & $31,2 \pm 5,14$ \\
\hline Contra-movimento $(\mathrm{cm})$ & $34,4 \pm 2,82$ & $35,13 \pm 4,58$ \\
\hline Salto pliométrico $(\mathrm{cm})$ & $32,64 \pm 6,51$ & $33,73 \pm 5,79$ \\
\hline
\end{tabular}

Tabela 4. Descrição das forças durante os saltos.

\begin{tabular}{|c|c|c|c|c|c|c|c|c|}
\hline \multirow{3}{*}{ Membro } & \multicolumn{8}{|c|}{ Força (N) } \\
\hline & \multicolumn{4}{|c|}{ Sub 15} & \multicolumn{4}{|c|}{ Sub 17} \\
\hline & Conc. & Exc. & $\begin{array}{l}\text { Imp. } \\
\text { inicial }\end{array}$ & Imp. final & Conc. & Exc. & $\begin{array}{l}\text { Imp. } \\
\text { inicial }\end{array}$ & $\begin{array}{l}\text { Imp. } \\
\text { final }\end{array}$ \\
\hline
\end{tabular}

\section{Salto em agachamento}

\begin{tabular}{c|c|c|c|c|c|c|c|c}
\hline Direito & $\begin{array}{c}792,55 \\
(61,48)\end{array}$ & - & - & $\begin{array}{c}2186,2 \\
(471,27)\end{array}$ & $\begin{array}{c}848,84 \\
(112,54)\end{array}$ & - & - & $\begin{array}{l}2356,65 \\
(270,45)\end{array}$ \\
\hline Esquerdo & $\begin{array}{c}868,89 \\
(303,51)\end{array}$ & - & - & $\begin{array}{c}1776,71 \\
(563,82)^{\mathrm{b}}\end{array}$ & $\begin{array}{c}949,49 \\
(534,64)\end{array}$ & - & - & $\begin{array}{l}(52,89 \\
(520,4)^{\mathrm{b}}\end{array}$ \\
\hline
\end{tabular}

\section{Contramovimento}

\begin{tabular}{c|c|c|c|c|c|c|c|c}
\hline Direito & $\begin{array}{c}802,32 \\
(103,19)\end{array}$ & $\begin{array}{c}823,34 \\
(207,01)\end{array}$ & - & $\begin{array}{c}2270,89 \\
(442,47)^{\mathrm{a}}\end{array}$ & $\begin{array}{c}903,31 \\
(137,98)\end{array}$ & $\begin{array}{c}925,67 \\
(165,55)\end{array}$ & - & $\begin{array}{c}2395,41 \\
(449,11)^{\mathrm{a}}\end{array}$ \\
\hline Esquerdo & $\begin{array}{c}769,87 \\
(92,48)\end{array}$ & $\begin{array}{c}807,22 \\
(209,75)\end{array}$ & - & $\begin{array}{c}2128,99 \\
(358,23)^{\mathrm{a}, \mathrm{b}}\end{array}$ & $\begin{array}{c}944,73 \\
(486,89)\end{array}$ & $\begin{array}{c}859,43 \\
(241,39)\end{array}$ & - & $\begin{array}{c}2065,3 \\
(552,24)^{\mathrm{a}, \mathrm{b}}\end{array}$ \\
\hline
\end{tabular}

\section{Salto pliométrico}

\begin{tabular}{c|c|c|c|c|c|c|c|c}
\hline Direito & - & - & $\begin{array}{c}1305,24 \\
(245,77)\end{array}$ & $\begin{array}{c}2216,39 \\
(447,25)\end{array}$ & - & - & $\begin{array}{c}1084,51 \\
(231,85)\end{array}$ & $\begin{array}{c}2221,35 \\
(455,82)\end{array}$ \\
\hline Esquerdo & - & - & $\begin{array}{c}922,11 \\
(270,25)\end{array}$ & $\begin{array}{c}1949,49 \\
(453,44)^{\mathrm{b}}\end{array}$ & - & - & $\begin{array}{c}890,19 \\
(208,61)\end{array}$ & $\begin{array}{c}2205,54 \\
(464,38)^{\mathrm{b}}\end{array}$ \\
\hline
\end{tabular}

Dados apresentados na forma de média (desvio padrão). ${ }^{a}$ indica diferença estatisticamente significativa em relaçăo ao salto squat jump. ${ }^{b}$ indica diferença estatisticamente significativa em relação ao membro direito.

Tabela 5. Razão de saltos contra-movimento por squat-jump para força concêntrica e altura do salto.

\begin{tabular}{c|c|c|c}
\hline \multirow{2}{*}{ Categorias } & \multirow{2}{*}{ Salto } & \multicolumn{2}{|c}{ Força concêntrica } \\
\cline { 3 - 4 } & & Direito & Esquerdo \\
\hline Sub 15 & $1,1(0,11)$ & $1,01(0,09)$ & $0,94(0,18)$ \\
\hline Sub 17 & $1,13(0,08)$ & $1,07(0,11)$ & $1,08(0,45)$ \\
\hline
\end{tabular}

Dados apresentados na forma de média (desvio padrão). 
Tabela 6. Correlação entre razão de saltos contra-movimento por salto em agachamento de altura de salto e força concêntrica em ambos os membros.

\begin{tabular}{c|c|c}
\hline \multirow{2}{*}{ Categorias } & \multicolumn{2}{|c}{ Força concêntrica } \\
\cline { 2 - 3 } & Direito & Esquerdo \\
\hline Salto Sub 15 & $\begin{array}{c}-0,06[-0,55 ; 0,47] \\
p=0,8258\end{array}$ & $\begin{array}{c}-0,17[-0,63 ; 0,37] \\
p=0,5326\end{array}$ \\
\hline Salto Sub 17 & $\begin{array}{c}-0,47[-0,79 ; 0,05] \\
p=0,0721\end{array}$ & $\begin{array}{c}-0,16[-0,62 ; 0,37] \\
p=0,5450\end{array}$ \\
\hline
\end{tabular}

Dados apresentados na forma de correlação [intervalo de confiança a 95\%], bem como p-valor.

Tabela 7. Descrição do déficit de força contra-lateral nos diferentes saltos e momentos

\begin{tabular}{c|c|c|c|c|c|c|c|c}
\hline \multirow{2}{*}{ Saltos } & \multicolumn{7}{c}{ Déficit força } \\
\cline { 2 - 9 } & \multicolumn{7}{c|}{ Sub 15 } & \multicolumn{3}{c}{ Sub 17 } \\
\cline { 2 - 9 } & Conc. & Exc. & $\begin{array}{c}\text { Imp. } \\
\text { inicial }\end{array}$ & $\begin{array}{c}\text { Imp. } \\
\text { Final }\end{array}$ & Conc. & Exc. & $\begin{array}{c}\text { Imp. } \\
\text { inicial }\end{array}$ & $\begin{array}{c}\text { Imp. } \\
\text { final }\end{array}$ \\
\hline $\begin{array}{c}\text { Squat- } \\
\text { jump }\end{array}$ & $\begin{array}{c}9,89 \\
(38,52)\end{array}$ & - & - & $\begin{array}{c}-17,95 \\
(21,7)\end{array}$ & $\begin{array}{c}9,63 \\
(48,8)\end{array}$ & - & - & $\begin{array}{c}-20,45 \\
(21,59)\end{array}$ \\
\hline $\begin{array}{c}\text { Contra- } \\
\text { movimento }\end{array}$ & $-3,52$ \\
$(8,62)$ & 3,62 & $(39,63)$ & - & $\begin{array}{c}-4,28 \\
(17,65)\end{array}$ & $\begin{array}{c}(3,6 \\
(36,41)\end{array}$ & $\begin{array}{c}-2,6 \\
(42,15)\end{array}$ & - & $\begin{array}{c}-9,53 \\
(35,89)\end{array}$ \\
\hline \multirow{2}{*}{\begin{tabular}{c} 
Drop-jump \\
\cline { 2 - 9 }
\end{tabular}} & - & - & $\begin{array}{c}-27,55 \\
(23,1)\end{array}$ & $\begin{array}{c}-11,78 \\
(11,35)\end{array}$ & - & - & $-14,2$ & 2,77 \\
$(25,5)$ & $(32,97)$ \\
\hline
\end{tabular}

Dados apresentados na forma de média (desvio padrão).

\section{DISCUSSÃO}

Avaliamos de forma única as capacidades funcionais e variáveis neurofuncionais de atletas de futebol de categoria de base durante a realização de três tipos de saltos verticais diferentes. Foi abrangida uma faixa etária na fase da puberdade, o que se deve ter em mente, pois estão em constante desenvolvimento das características neuromusculares de forças ${ }^{19,20}$. Esse tipo de estudo de avaliação de saltos verticais através de plataformas de força vem para complementar e incrementar a área da musculoesquelética com pesquisa instrumentada, mostrando-se eficaz para fazer uma leitura dos gestos dos saltos, ativação neuromuscular e resposta mecânica $a^{21,22}$.

Houve acréscimo de peso e estatura com o aumento da idade, observando ligeira mudança de somente uma categoria (sub-15 para a sub-17). Não se pode concluir ou inferir que esse ganho de peso se transmita diretamente para uma maior capacidade de força da categoria sub-17. No desempenho da altura dos saltos houve a esperada melhora do contramovimento e do drop jump em relação ao squat jump. Devido à teoria do aproveitamento da força inicial, é possível saltar mais alto no contramovimento (tem movimento de preparação) do que o squat jump $p^{23}$.

Pode-se notar também ganho na altura do salto da categoria sub-17 em relação à categoria sub-15 (exceto no squat jump), porém não de forma estatisticamente significativa. Um achado que vai de encontro à literatura ${ }^{23,24}$ foi o melhor desempenho no contramovimento em relação ao drop jump. Mas deve-se levar em consideração que não foi da mesma forma estatisticamente significante. A diferença entre o rendimento máximo no drop jump e no contramovimento é o critério para o aproveitamento do mecanismo no ciclo de estiramento-encurtamento ${ }^{23}$. Essa diferença pode ser explicada pelo fato de os atletas não estarem familiarizados com os tipos de salto ${ }^{20} \mathrm{e}$ a altura escolhida de $40 \mathrm{~cm}$ não ser considerada ideal para a população estudada, apesar de a literatura indicá-la como satisfatória23,25.

De acordo com os resultados apresentados para as descrições das forças analisadas nos saltos, não foi evidenciada diferença no padrão de força concêntrica entre categorias, membros ou saltos, mostrando uma boa mecânica do salto, controle entre os membros e leve influência da idade devido ao maior valor bruto (não significante) para a categoria sub-17. Achados da literatura confirmam essa possível evolução com a idade e melhor desempenho nos saltos ${ }^{19}$. Quando comparamos a distribuição de força no impacto final para os três tipos de saltos, observou-se diferença significativa no impacto do salto contramovimento em relação ao squat jump independente da categoria etária e membro considerado; além disso, a diferença entre membro esquerdo e direito foi significativa, desconsiderando tipo de salto e categoria etária. Esse achado é confirmado na tabela 7, que mostra a diminuição dos valores para o membro inferior esquerdo, e por não ser estatisticamente significativo não corresponde a algum déficit de força ipsilateral.

Essa última questão abordada sobre a diferença entre as forças de impacto pode ser devida à maior altura alcançada após fase de preparação (ciclo encurtamento-alongamento), que não se encontra no squat jump, que por reação implica numa aterrissagem maior. Esse aumento do input neural, em consequência do reflexo de estiramento, na fase inicial do salto, ao ter contato inicial com o solo, pode ser inibido devido ao excesso de impacto ou à fadiga neural ou metabólica, dissipando a energia acumulada. A velocidade ideal de movimento para a fase concêntrica $(0,2 \mathrm{~m} / \mathrm{s})$ e para a fase excêntrica $(0,3 \mathrm{~m} / \mathrm{s})$ também sofre interferência ${ }^{23}$. Déficit do ciclo encurtamento-alongamento pode demonstrar perda de potência muscular, queda no desempenho em atividades funcionais que exijam mecanismo pliométrico, maior descarga no membro inferior contralateral ou melhor utilização desse ciclo no membro contralateral24.

Maior descarga de peso na fase de impacto pode indicar uma má desaceleração do movimento, sem a devida flexão dos joelhos e, assim, acarretar em problemas articulares nos tornozelos e, principalmente, nos joelhos. Mecanismo de proteção do membro inferior contralateral também pode estar relacionado. No caso das mulheres, há uma relação com o valgismo anatômico do joelho e a propensão de lesão não impactada do ligamento cruzado anterior (LCA $)^{26}$. Com a execução dos saltos, é natural que o indivíduo tende a realizar um valgo de joelhos na aterrissagem, sendo um aspecto que se deve ter em vista e alertar para que o indivíduo controle o movimento durante todo o salto, principalmente na queda ${ }^{27}$.

Foi verificado que o uso do salto pliométrico como treinamento aumenta o alinhamento dos joelhos e tornozelos em mulheres, a estabilização das articulações e ganho estatisticamente significante na média do pico de torque flexor dos joelhos ${ }^{27}$. O treinamento e a experiência de modo geral implicam em uma maior ação motora e, por consequência, respostas posturais, pois o desenvolvimento do desempenho sensório-motor é estímulo-dependente ${ }^{14,28}$. Isso pode ser transferido para uma melhora da capacidade de realização dos saltos, principalmente na absorção do impacto na aterrissagem final, e/ou reutilização de energia acumulada após o primeiro impacto para a fase concêntrica e de voo do salto. Também pode controlar melhor o corpo em todas as fases dos saltos.

Da mesma forma que o melhor controle do corpo pode inferir em um melhor desempenho do salto (referente a mecânica realizada), Davis et al., mostraram que indivíduos com maior capacidade de equilíbrio tendem a ter pior desempenho nos saltos verticais. Isso pode ser explicado pela possível relação com os tipos de fibra muscular I e II, tendo o tipo | função primária de estabilização postural, e o tipo || correspondente a movimentos rápidos, força explosiva. Bem como a maior força excêntrica do quadríceps correlacionou-se positivamente com maior altura no salto. O que se pode confirmar comparando ao nosso estudo, pois mesmo não sendo estatisticamente significativo, 
houve aumento na altura do salto contramovimento no sub-17 em relação ao sub-15 e sendo confirmada com a maior força excêntrica encontrada nesse salto ${ }^{14}$.

Não foi possível estabelecer uma correlação entre a razão de alteração da altura do salto e a alteração na aplicação da força concêntrica em ambos os membros. Tal resultado corrobora o fato de que a força concêntrica não difere de forma estatisticamente significativa entre os saltos analisados. Essa razão só confirma a evolução do desempenho com a idade e o maior rendimento do contramovimento em relação ao squat jump, mesmo que não sendo significante. É interessante ter isso em prática pela relação existente entre a força máxima no squat jump e o desempenho na capacidade explosiva e altura do salto. Sendo transferido o treinamento realizado em saltos verticais, de caráter explosivo, para um arranque ou atividade que exija contração rápida da musculatura sem altos consumos de oxigênio. Assim, implica em uma ênfase na fase concêntrica da musculatura ${ }^{19,20,29}$.

Outras variáveis que não são absolutamente controladas como tipo de calçado, tipo de pé, sobrecarga mecânica do treinamento e controle postural já foram descritas na literatura ${ }^{23}$.

\section{REFERÊNCIAS}

1. Cohen M, Ejnisman B. Futebol. Em: Cohen M, Abdalla RJ, editores. Lesões nos esportes., São Paulo: Revinter; 2003.

2. Junge A, Rosch D, Peterson L, Graf-Baumann T, Dvorak J. Prevention of soccer injuries: a prospective intervention study in youth amateur players. Am J Sports Med 2002;30:652-9.

3. Dvorak J, Junge A. Football injuries and physical symptoms: a review of the literature. Am J Sports Med 2000;28:S3-9.

4. Morgan B, Oberlander M. An examination of injuries in major league soccer. Am J Sports Med 2001;29:426-30.

5. Ekstrand J, Gillquist J, Moller M, Oberg B, Liljedahl SO. Incidence of soccer injuries and their relation to training and team success. Am J Sports Med 1983;11:63-7.

6. Engström B, Johansson C, Törnkvist H. Soccer injuries among elite female players. Am J Sports Med 1991;19:372-5.

7. Hägglund $M$, Waldén $M$, Ekstrand J. Previous injury as a risk factor for injury in elite football: a prospective study over two consecutive seasons. Br J Sports Med 2006;40:767-72.

8. Engebretsen AH. Prevention of injuries among male soccer players. A prospective, randomized intervention study targeting players with previous injuries or reduced function. Am J Sports Med 2008;36:1052-60.

9. Ekstrand J, Gillquist J, Liljedahl SO. Prevention of soccer injuries: supervision by doctor and physiotherapist. Am J Sports Med 1983;11:116-20.

10. Pataro S, Oliveira C, Souza A. Princípios da reabilitação proprioceptiva. Em: Souza A, editor. Propriocepção. Rio de Janeiro: Medsi; 2004.

11. Gallaspy JB. Reabilitação dos músculos isquiotibiais, do quadríceps e dos músculos da virilha. Em: Andrews JR, Harrelson GL, Wilk KE, editores. Reabilitação física das lesões desportivas. Rio de Janeiro: Guanabara Koogan; 2000.

12. Jennings CL, Viljoen W, Durandt J, Lambert MI. The reliability of the FitroDyne as a measure of muscle power. J Strength Condit Res 2005;19:859-63.

13. Young W, Wilson G, Byrne C. Relationship between strength qualities and performance in standing and run-up vertical jumps. J Sports Med Phys Fitness 1999;39:285-93.

14. Davis DS, Briscoe DA, Markowski CT, Saville SE, Taylor CJ. Physical characteristics that predict vertical jump performance in recreational male athletes. Phys Ther Sports 2003;4:167-74

15. Weber MD, Ware AN. Reabilitação do joelho. Em: Andrews JR, Harrelson GL, Wilk KE, editores. Reabili-

\section{CONCLUSÃO}

Encontramos uma assimetria entre os membros inferiores, podendo correlacionar com um déficit de potência muscular, má desaceleração do movimento e erros biomecânicos. Fatores predisponentes a lesões musculoesqueléticas, os quais podem ser prevenidos. Houve também o esperado avanço com o aumento da idade, porém indica-se que avaliações com outras faixas etárias sejam feitas para corroborar esses resultados, tornando-os estatisticamente significantes. A avaliação instrumentada da força através desses testes pode subsidiar a equipe médica e técnica do clube como uma ferramenta de trabalho, capaz de aprimorar programas de treinamento, preparação e reabilitação. Entretanto, são necessárias mais pesquisas na área, principalmente um acompanhamento longitudinal das categorias, com avaliações pré-programadas de forma periódica e até durante os treinamentos, verificando evoluções e até involuções com o modo de trabalho imposto.

Todos os autores declararam não haver qualquer potencial conflito de interesses referente a este artigo. tação física das lesões desportivas. Rio de Janeiro: Guanabara Koogan; 2000.

16. Urquiza MA, Soares AB, Pereira AA, Sousa JV. Plataforma de força tridimensional para análises biomecânicas e cinesiológicas. Horizonte Científico 2007;1.

17. Bizzo G, Guillet N, Patat A, Gagey PM. Specifications for building a vertical force platform designed for clinical stabilometry. Med Biol Eng Comput 1985;23:474-6.

18. Cappello A, Lenzi D, Chiari L. Periodical in-situ re-calibration of force platforms: a new method for the robust estimation of the calibration matrix. Med Biol Eng Comput 2004;42:350-5.

19. Paasuke M, Ereline J, Gapeyena H. Knee extensor muscle stregth and vertical jumping performance characteristics in pré- and post-pubertal boys. Pediat Exerc Sci 2001;13:60-9.

20. Pereira R, Machado M, Santos MM, Pereira LN, Sampaio-Jorge F. Muscle activation sequence compromises vertical jump performance. Serb J Sports Sci 2008;2:85-90.

21. Bosco C, Luhtanen P, Komi PV. A simple method for measurement of mechanical power in jumping Eur J Appl Physiol 1982;50:273-82.

22. Bisciotti GN. II salto sotto esame. Sport\&Med 2004;51-7.

23. Amadio AC, Avila AOV, Guimarães ACS, David AC, Mota CB, Borges DM, et al. Métodos de medição em biomecânica do esporte: descrição de protocolos para aplicação nos centros de excelência esportiva (rede CENESP - MET). Rev Bras Biomecanic 2002;3:57-67.

24. Bosco C. Aspectos Fisiológicos de La Preparación Física Del Futbolista. Deporte \& Entrenamiento Colección Fútbol. 3a ed. Barcelona: Paidotribo; 1996.

25. Bosco C. Propuesta de valoración de lãs capacidades fisiológicas y neuromusculares Del futebolista. Em: Bosco C. Fisiológicos de La Preparación Física Del Futbolista. Barcelona: Paidotribo; 1996.

26. Berns GS, Hull ML, Patterson HA. Strain in the anteromedial bundle of the anterior cruciate ligament under combination loading. J Orthop Res 1992;10:167-76.

27. Noyes FR, Barber-Westin SD, Fleckenstein C, Walsh C, West J. The drop-jump screening test: difference in lower limb control by gender and effect of neuromuscular training in female athletes. Am J Sports Med 2005;33:197-207.

28. Yoshitomi SK, Tanaka C, Duarte M, Lima F, Morya E, Hazime F. Respostas posturais à perturbação externa inesperada em judocas de diferentes níveis de habilidade. Rev Bras Med Esporte 2006;12:159-63.

29. Wisloff U, Castagna C, Helgerud J, Jones R, Hoff J. Strong correlation of maximal squat strenght with sprint performance and vertical jump height in elite soccer players. Br J Sports Med 2004;38:285-8. 